\title{
Sinusoidal, Pulse, Triangular Oscillator Using Second Generation Current Conveyor
}

\author{
Jin-Ho Choi, Member, KIMICS
}

\begin{abstract}
This paper describes the sinusoidal, pulse, triangular oscillator using second generation current conveyor. To obtain the sinusoidal waveform the circuit blocks are constructed by using all pass filter and integrator. The pulse and the triangular waveforms are obtained from the output of sinusoidal oscillator. The peak-to-peak voltages of sinusoidal and triangular waveforms can be easily controlled by the dc offset voltage. Also the output frequency of the oscillator can be controlled by varying passive elements. The designed circuit is verified by HSPICE simulation.
\end{abstract}

Index Terms - sinusoidal oscillator, pulse oscillator, triangular oscillator, second generation current conveyor, operational amplifier.

\section{INTRODUCTION}

The second generation current conveyor(CCII) was introduced by Sedra and Smith in 1970[1]. The CCII has many advantages such as lower power consumption, better linearity and stability properties. Therefore CCII has been found useful in many applications and a number of schemes have been developed to realize the filters and oscillators[2-5]. Many application circuit blocks can be implemented by using CCIIs easier than using operational amplifier because the operation of CCII approaches its ideal behavior quite closely. CCII is available with both polarities as positive(CCII + ) and negative(CCII-). CCII using integrated circuit such as LM741 was represented by S. Rana and K. Pal in 2007[6]. The circuit realized both positive and negative polarities CCIIs. In this paper the sinusoidal, pulse, triangular oscillators are introduced. The circuits are based on CCII- using operational amplifier. In the CCII+ circuit the current supplied to input node is conveyed to output node and in the CCII- circuit the output current direction is reverse compared to the output current direction in the CCII+.

In section II, the structure and the operation of CCII circuit are described. In section III, the designed sinusoidal, pulse, triangular oscillators are introduced.

\footnotetext{
Manuscript received August 27, 2010; revised September 11, 2010; accepted September 17, 2010.

Jin-Ho Choi is with the Department of Embedded IT, Pusan University of Foreign Studies, Busan, 608-738, Korea (Email: jhchoi@pufs.ac.kr)
}

Also the operation of the oscillators is analyzed using HSPICE simulation tool. In section IV, the characteristics of the oscillators are described and finally, the conclusions show in Section V.

\section{CURRENT CONVEYOR CIRCUIT}

Fig. 1 shows the CCII+ circuit symbol with positive polarity, and the ideal current conveyer can be described by the following equations (1), (2) and (3). When a voltage is applied at node $y$, that voltage is transferred to node $\mathrm{x}$. This is similar to virtual short on an operational amplifier. The current supplied to node $\mathrm{x}$ is conveyed to node $\mathrm{z}$.

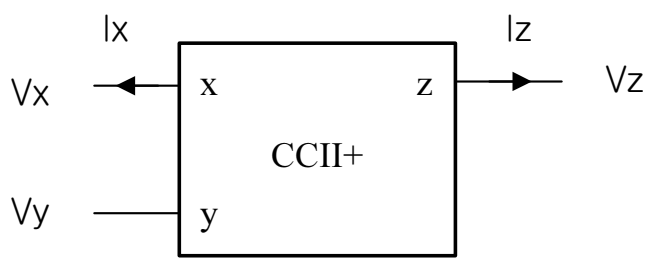

Fig. 1 Circuit symbol of the CCII+

$$
\begin{aligned}
& i_{y}=0 \\
& v_{x}=v_{y} \\
& i_{z}=i_{x}
\end{aligned}
$$

Fig. 2 shows the CCII- circuit symbol with negative polarity. In CCII- the current direction at the $\mathrm{Z}$ node is reverse compared to the current direction in the CCII+. Therefore the current equation is expressed by equation (4).

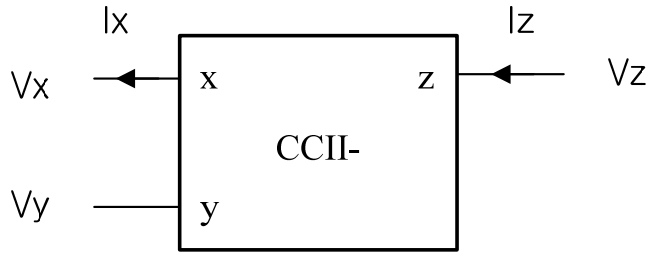

Fig. 2 Circuit symbol of the CCII- 


$$
i_{z}=-i_{x}
$$

Fig. 3 shows the CCII- circuit using operational amplifier[6]. The circuit was proposed by S. Rana and $\mathrm{K}$. Pal. The input node voltages, $\mathrm{V}_{\mathrm{x}}$ and $\mathrm{V}_{\mathrm{y}}$ are the same. The current $i_{x}$ flowing through the resistor is expressed as equation (5) because the input current is nearly zero.

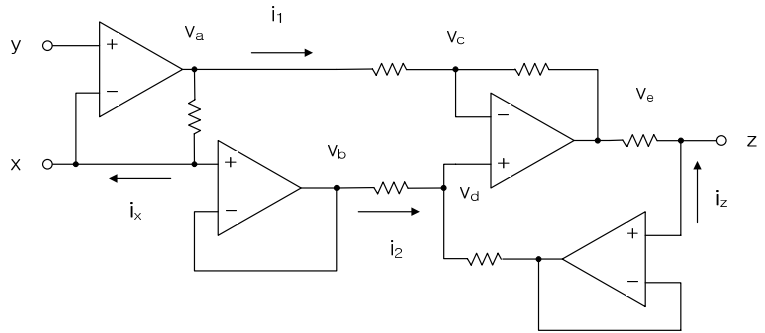

Fig. 3 Second generation current conveyor

$$
i_{x}=\frac{V a-V b}{R}
$$

The current $i_{1}$ can be given by

$$
i_{1}=\frac{V a-V c}{R}=\frac{V c-V e}{R}
$$

From equation (6) $\mathrm{Vc}$ is expressed by

$$
2 V c=V a+V e
$$

From the similar method the current $i_{2}$ can be given by

$$
i_{2}=\frac{V b-V d}{R}=\frac{V d-V z}{R}
$$

From equation (8) Vd is expressed by

$$
2 V d=V b+V z
$$

$\mathrm{Vd}$ is equal to $\mathrm{Vc}$ because the voltages are the same in inverting node and non-invertring node of operational amplifier.

From equation (7) and equation (9) the equation is rearranged as follows:

$$
\frac{V e-V z}{R}=\frac{V b-V a}{R}
$$

Solving for the currents $i_{x}$ and $i_{z}$, it thus results in

$$
i_{z}=-i_{x}
$$

Fig. 4 shows the basic structure of CMOS second generation current conveyor circuit. The designed circuit is based on operational amplifier CCII.

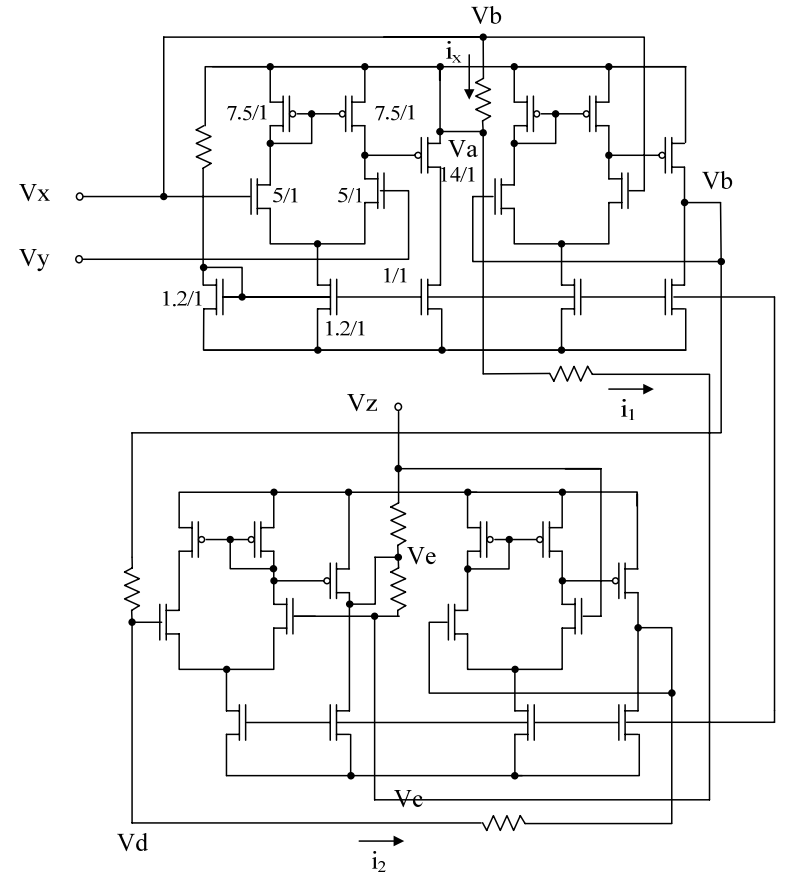

Fig. 4 CMOS Second generation current conveyor

\section{SINUSOIDAL, PULSE, TRIANGULAR GENERATORS}

Fig 5 shows the circuit of sinusoidal, pulse, triangular oscillators. The all pass filter circuit is realized using $\mathrm{CCII}_{1^{-}}, \mathrm{C}_{1}, \mathrm{R}_{1}, \mathrm{C}_{2}$ and $\mathrm{R}_{2}$. And the integrator is realized $\mathrm{CCII}_{2^{-}}, \mathrm{C}_{3}$ and $\mathrm{R}_{3}$. In Fig. 4 the all-pass filter circuit was introduced by P. Kumar and K. Pal in 2008[7]. In the $\mathrm{CCII}_{1}$ - the voltage transfer function is given by equation (12)

$$
\frac{V_{2}}{V_{1}}=\frac{1+s^{2} C_{1} C_{2} R_{1} R_{2}+s\left(C_{1} R_{1}+C_{2} R_{2}-C_{1} R_{2}\right)}{1+s^{2} C_{1} C_{2} R_{1} R_{2}+s\left(C_{1} R_{1}+C_{2} R_{2}\right)}
$$

For all-pass function the magnitude of $\mathrm{V}_{2} / \mathrm{V}_{1}$ have to equal one. The relation of $\mathrm{C}_{1}, \mathrm{C}_{2}, \mathrm{R}_{1}$ and $\mathrm{R}_{2}$ is expressed by equation (13) and the condition is satisfied when $\mathrm{R}_{2}=4 \mathrm{R}_{1}$ and $\mathrm{C}_{1}=4 \mathrm{C}_{2}$.

$$
R_{1}=\frac{R_{2}\left(C_{1}-2 C_{2}\right)}{2 C_{1}}
$$

From equation (13) the parameter $\omega 0$ can be expressed as

$$
\omega_{o}=\frac{1}{R_{1} C_{1}}=\frac{1}{R_{2} C_{2}}
$$

In the $\mathrm{CCII}_{2}$ - the voltage transfer function is express by 


$$
\frac{V_{3}}{V_{2}}=\frac{1}{s R_{3} C_{3}}
$$

For sinusoidal oscillations loop gain, $\mathrm{V}_{3} / \mathrm{V}_{1}$, is one. And the parameter $\omega o$ can be expressed as

$$
\varpi_{o}=\frac{1}{R_{3} C_{3}}
$$

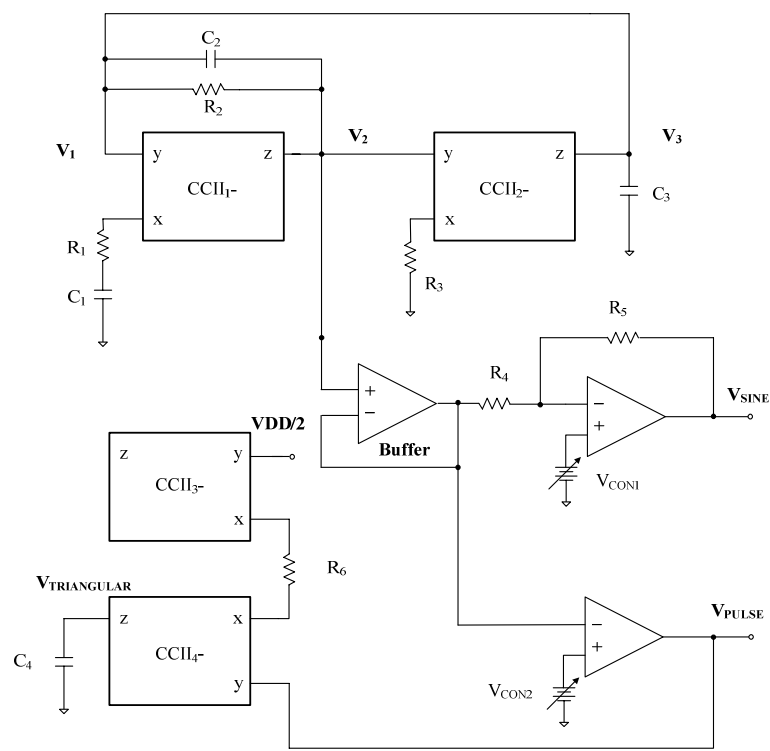

Fig. 5 Circuit diagram of sinusoidal, pulse, triangular oscillators.

In Fig. 5 the buffer is used to cancel the loading effect. To control the amplitude and offset of the sinusoidal waveform the operational amplifier is used. $\mathrm{V}_{\mathrm{CON} 1}$ is the offset control voltage and the output voltage swing range is controlled by controlling resistances R4 and R5. To obtain the pulse waveform the buffer output is compared with $\mathrm{V}_{\mathrm{CON} 2}$. Also the duty of the pulse output can be controlled by varying $\mathrm{V}_{\mathrm{CON} 2}$. The triangular waveform generator is obtained from the $\mathrm{CCII}_{3-}$ and the $\mathrm{CCII}_{4^{-}}$. The capacitor, $\mathrm{C} 4$, is discharged when $\mathrm{V}_{\mathrm{PULSE}}$ is $\mathrm{V}_{\mathrm{DD}}$ and the discharge current is as follows:

$$
i_{\text {discharge }}=\frac{V_{D D}}{2 R_{6}}
$$

The capacitor is charged when $V_{\text {PULSE }}$ is zero and the charge and discharge currents are the same. The peak-topeak voltage of the triangular waveform is expressed by

$$
V_{P E A K-t o-P E A K}=\frac{T}{C_{4}} \frac{V_{D D}}{2 R_{6}}=\frac{1}{f_{o} C_{4}} \frac{V_{D D}}{R_{6}}
$$

where $T$ and $f_{o}$ are the period and frequency of the sinusoidal waveform, respectively. From equation (18) the peak-to-peak voltage of the triangular waveform can be controlled by varying $\mathrm{C}_{4}$ or $\mathrm{R}_{6}$.

Fig. 6 shows the outputs of sinusoidal, pulse and triangular oscillators. Fig. 6(a) shows $\mathrm{V}_{\mathrm{SINE}}$ waveform. Fig. 6(b) and Fig. 6(c) show $V_{\text {PULSE }}$ and V V Trangular waveforms.

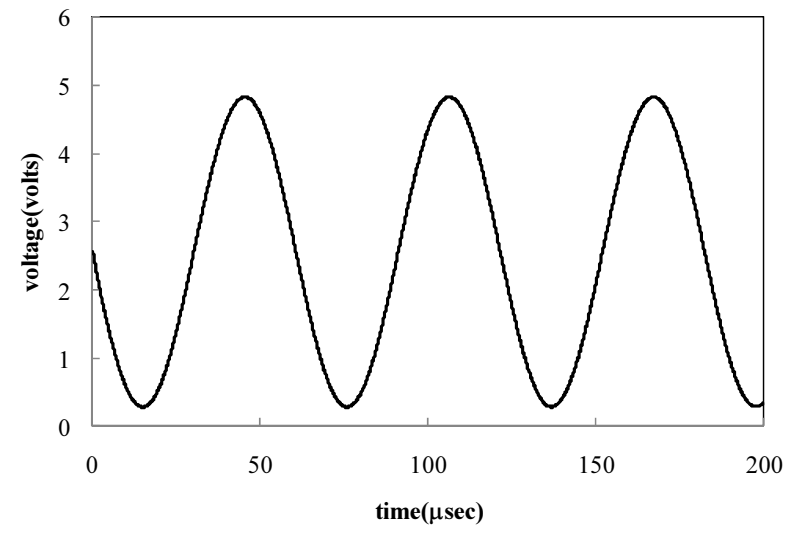

(a)

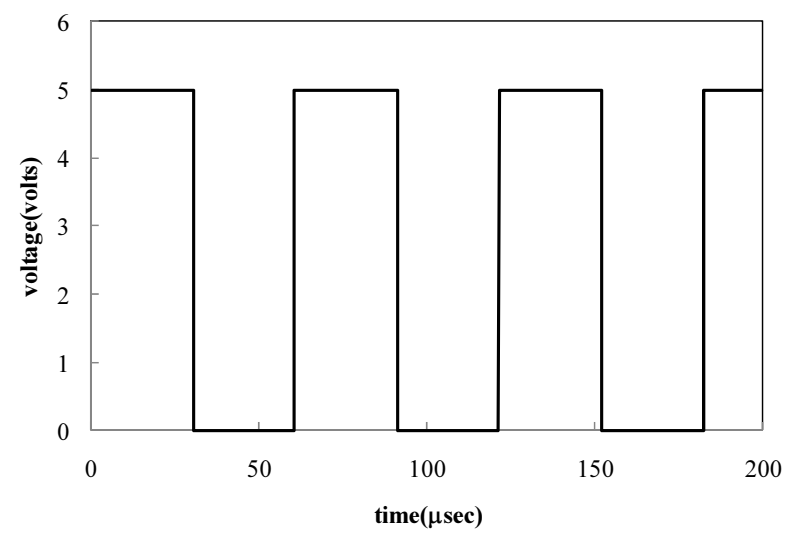

(b)

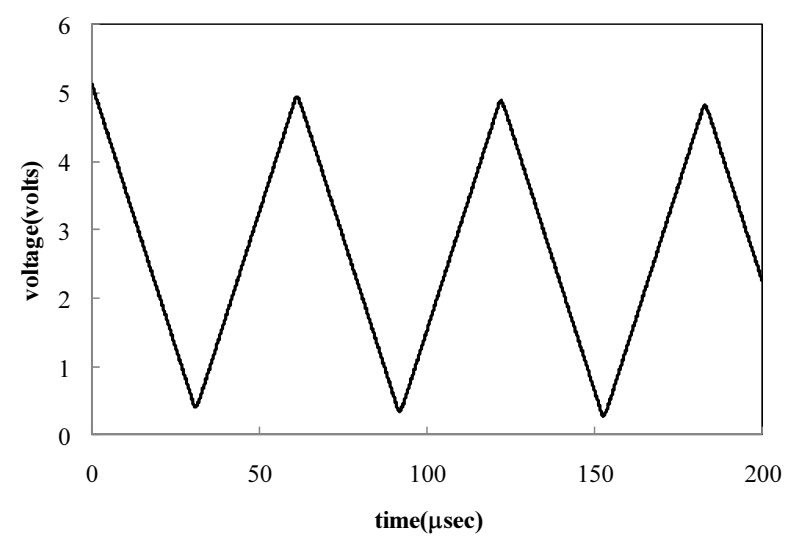

(c)

Fig. 6 The output waveforms (a) sinusoidal generator (b) pulse generator $(c)$ triangular generator 


\section{ANALYSIS OF SINUSOIDAL, PULSE, TRIANGULAR GENERATORS}

Fig. 7(a) shows the variation of frequency with $\mathrm{R}_{3} \mathrm{C}_{3}$. Fig. 7(b) shows the error with $\mathrm{R}_{3} \mathrm{C}_{3}$. The error is calculated as follows:

$$
\operatorname{error}(\%)=\frac{f_{\text {calculation }}-f_{\text {simulation }}}{f_{\text {calculation }}} \times 100
$$

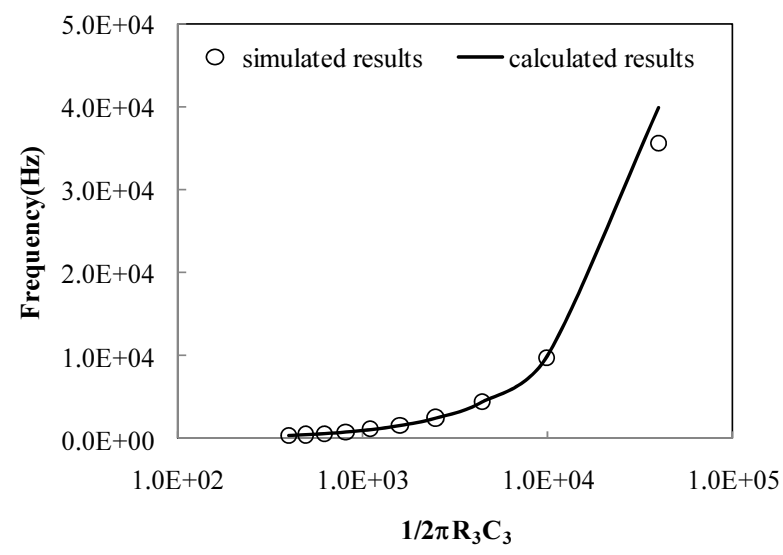

(a)

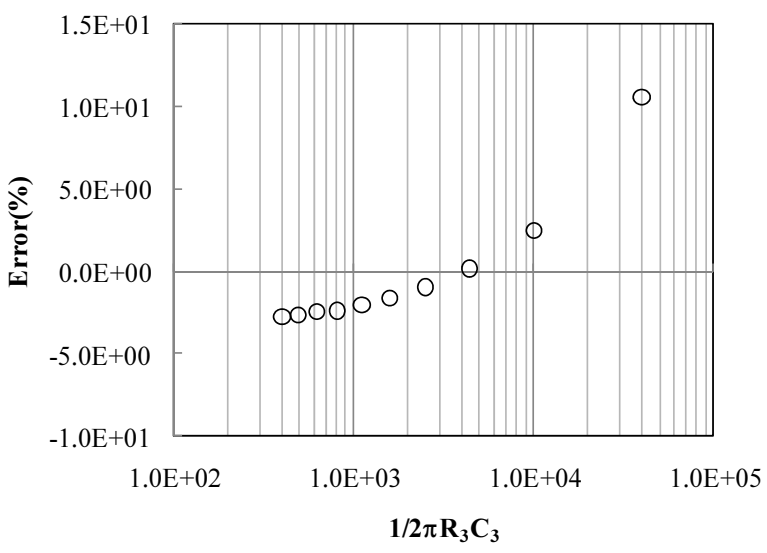

(b)

Fig. 7 Simulation results with $\mathrm{R}_{3} \mathrm{C}_{3}$ (a) frequency variation with $\mathrm{R}_{3} \mathrm{C}_{3}$ (b) error with $\mathrm{R}_{3} \mathrm{C}_{3}$

From the simulation results the error increases slightly with the frequency increases compared to calculated frequency. Therefore $\mathrm{R}_{3} \mathrm{C}_{3}$ should be controlled finely with the frequency increases.

\section{CONCLUSIONS}

The various signal oscillators are designed and the circuits are designed using the CCII-. The CCII circuit is based on operational amplifier. The output frequency can be easily controlled by varying the RC. From HSPICE simulation results the proposed circuit shows enough good characteristics in low frequency. But as increasing the frequency the value of $\mathrm{RC}$ has to decrease compared to the calculated $\mathrm{RC}$ value.

\section{REFERENCES}

[1] Sedra, A. and Smith K. C., "A Second Generation Current Conveyor and Its Applications", IEEE Trans. Circuit Theory, vol. 17, pp.132-134, 1970.

[2] Jiun-Wei Horng, Sheng-Feng Lin and Chao-Tang Yang, "Sinusoidal Oscillators Using Current Conveyors and Grounded Capacitors", J. of Active and Passive Electronic Devices, vol. 2, pp.127-136, 2007.

[3] Amphawan Julsereewong, "Differential Voltage-to-Frequency Converter for Telemetry", Proceedings of the International MultiConference of Engineerings and Computer Scientist, vol III, IMECS 2009.

[4] Khan, I. A. and Jaidi, H. Mehmood, "Multifunctional translinearC-current mode filter”, Int. J. Electronics, vol. 87, no. 9, pp. 10471051,2000

[5] Toker, A. and Ozoguz, S., "Insensitive Current Mode Universal Filter with Single input and Three Outputs", JEE, vol. 88, no. 3, pp.333-337, 2001.

[6] S. Rana and K. Pal, "Current Conveyor Simulation Circuits Using Operational Amplifiers", j. of Physical Sciences, vol. 11, pp. 124132, 2007.

[7] P. Kumar and K. Pal, "Universal Biquadratic Filter Using a Single Current Conveyor", J. of Active and Passive Electronic Devices, vol. 3, pp.7-16, 2008

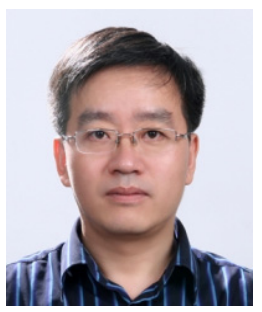

Jin-Ho Choi (M'85-SM'87-F'92) received the B.S. degree in electronics from Busan University in 1985, and the M.S. degree in electronics from KAIST in 1987. He received the Ph. D. degree in electronics from KAIST in 1992. From 1992 to 1996, he was a researcher at Hynix Semiconductor Inc.. Since 1996, he has been a professor in Dept. of Computer Engineering, Pusan University of Foreign Studies. His research interest includes VLSI design. 